Algebra Univers. 63 (2010) 283-301

DOI $10.1007 / \mathrm{s} 00012-010-0079-\mathrm{y}$

Published online September 8, 2010

(c) The Author(s) 2010.

Algebra Universalis

This article is published with open access at Springerlink.com

\title{
The $\mathcal{H S}=\mathcal{S H}$ problem for coalgebras
}

\section{Tomasz Brengos and VĚra Trnková}

\begin{abstract}
H. P. Gumm and T. Schröder stated a hypothesis that the preservation of preimages by a functor $T$ for which $|T 1|=1$ is equivalent to the satisfaction of the class equality $\mathcal{H} \mathcal{S}(\mathrm{K})=\mathcal{S H}(\mathrm{K})$ for any class $\mathrm{K}$ of $T$-coalgebras. Although we were not able to find a full solution, our paper gives a positive answer to this problem for a very wide class of Set-endofunctors.
\end{abstract}

\section{Introduction}

H. P. Gumm and T. Schröder [4] stated the following problem:

Is the satisfaction of the equation $\mathcal{H} \mathcal{S}(\mathrm{K})=\mathcal{S H}(\mathrm{K})$ for any class $\mathrm{K}$ of $T$-coalgebras equivalent to $T$ preserving preimages?

The authors conjectured a positive answer to this question. The conjecture is supported by the following two theorems.

Theorem $1.1([4])$. Assume that a functor $T:$ Set $\rightarrow$ Set preserves preimages. Then for any class $\mathrm{K}$ of $T$-coalgebras $\mathcal{H} \mathcal{S}(\mathrm{K})=\mathcal{S H}(\mathrm{K})$.

Theorem $1.2([4])$. Let $T$ : Set $\rightarrow$ Set be a functor such that $|T 1|>1$. If $\mathcal{H S}(\mathrm{K})=\mathcal{S H}(\mathrm{K})$ for any class $\mathrm{K}$ of $T$-coalgebras, then $T$ preserves preimages.

By Theorems 1.1 and 1.2 it follows that in order to find a positive answer to the $\mathcal{H S}=\mathcal{S H}$ problem it is enough to find, for any functor $T$ : Set $\rightarrow$ Set not preserving preimages and satisfying $|T 1|=1$, a $T$-coalgebra $\mathbb{X}$ such that $\mathcal{S H}(\mathbb{X}) \neq \mathcal{H S}(\mathbb{X})$. However, as it will be shown below, the constant functor $\mathcal{C}_{0,1}$ sending the empty set to itself and all non-empty sets to a one-element set is a counterexample. The condition which should be investigated to be equivalent to $\mathcal{H} \mathcal{S}(\mathrm{K})=\mathcal{S H}(\mathrm{K})$ is the preservation of non-empty preimages.

Presented by H. P. Gumm.

Received December 10, 2008; accepted in final form September 16, 2009.

2000 Mathematics Subject Classification: Primary: 03B70; Secondary: 03C99.

Key words and phrases: coalgebra, preimage preservation, functor, coalgebraic logic.

The first author was supported by the Grant of Warsaw University of Technology no. 504G 11200112000 and acknowledges the hospitality of the Faculty of Mathematics and Physics, Charles University, during his visit to Prague in September 2008, when a part of the work on the paper was completed. The second author gratefully acknowledges the support of MSM 0021620839, a project of the Czech Ministry of Education, and of the grant 201/06/0664 by the Grant Agency of Czech Republic. 
Any Set-endofunctor $T$ which does not preserve non-empty preimages and satisfies $|T 1|=1$ falls into exactly one of the two classes:

- functors which do not preserve non-empty preimages on undistinguished points,

- functors which preserve non-empty preimages on undistinguished points.

In the first part of this article we present a construction of a $T$-coalgebra $\mathbb{X}$ with $\mathcal{S H}(\mathbb{X}) \neq \mathcal{H} \mathcal{S}(\mathbb{X})$ for any functor $T$ not preserving non-empty preimages on undistinguished points.

The second part focuses on finding a $T$-coalgebra $\mathbb{X}$ that fails to satisfy $\mathcal{S H}(\mathbb{X})=\mathcal{H S}(\mathbb{X})$ for a functor $T$ not preserving non-empty preimages but preserving them on undistinguished points. In this case, at the time of writing the paper we were not able to give a complete solution to the problem. Instead, we present a general setting in which we are able to construct a coalgebra $\mathbb{X}$ with $\mathcal{S H}(\mathbb{X}) \neq \mathcal{H} \mathcal{S}(\mathbb{X})$ and give examples of families of functors that can be put in the setting.

\section{Basic notions}

Let Set be the category of all sets and mappings between them. Let $T:$ Set $\rightarrow$ Set be a functor. A $T$-coalgebra $\mathbb{X}$ is a pair $\langle X, \xi\rangle$, where $X$ is a set and $\xi$ is a mapping $\xi: X \rightarrow T X$. The set $X$ is called a carrier and the mapping $\xi$ is called a structure of the coalgebra $\mathbb{X}=\langle X, \xi\rangle$.

A homomorphism from a $T$-coalgebra $\mathbb{X}=\langle X, \xi\rangle$ to a $T$-coalgebra $\mathbb{Y}=$ $\langle Y, \psi\rangle$ is a mapping $h: X \rightarrow Y$ such that $T(h) \circ \xi=\psi \circ h$.

A $T$-coalgebra $\mathbb{S}=\langle S, \sigma\rangle$ is said to be a subcoalgebra of a $T$-coalgebra $\mathbb{X}=\langle X, \xi\rangle$ whenever there is an injective homomorphism from $\mathbb{S}$ into $\mathbb{X}$. This fact is denoted by $\mathbb{S} \leq \mathbb{X}$.

Let $h: \mathbb{X} \rightarrow \mathbb{Y}$ be a homomorphism and let $\mathbb{S} \leq \mathbb{X}$. Then there is a structure $\delta: h(S) \rightarrow T(h(S))$ such that $h_{\mid S}: S \rightarrow h(S)$ is a homomorphism from $\mathbb{S}=$ $\langle S, \sigma\rangle$ onto $\langle h(S), \delta\rangle$ and $\langle h(S), \delta\rangle \leq\langle Y, \psi\rangle$. In other words, a homomorphic image of a subcoalgebra of a domain of $h$ is a subcoalgebra of a codomain of $h$. For the basics on the theory of coalgebras the reader is referred to [2].

A functor $T$ : Set $\rightarrow$ Set preserves preimages if for any mapping $f: A \rightarrow B$ and a subset $C \subset B$ the following diagram is a pullback diagram.

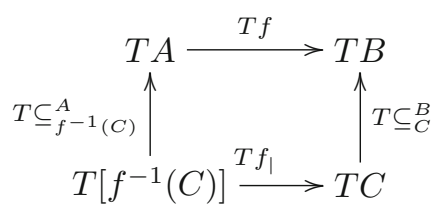

In other words, a functor $T:$ Set $\rightarrow$ Set does not preserve preimages whenever there is a mapping $f: A \rightarrow B$ and a subset $C \subset B$ with $\vec{a} \in T A$ and $\vec{c} \in T C$ such that $[T f](\vec{a})=\left[T \subseteq{ }_{C}^{B}\right](\vec{c})$ and the element $\vec{a}$ does not belong to the image of the set $T\left[f^{-1}(C)\right]$ under the map $T\left[\subseteq_{f^{-1}(C)}^{A}\right]$. 
Let $\mathcal{C}_{0,1}$ denote the constant functor for which $\mathcal{C}_{0,1} \varnothing=\varnothing$ and $\mathcal{C}_{0,1} A=\{\perp\}$ for $A \neq \varnothing$ and for which given $f: A \rightarrow B$ the map $\mathcal{C}_{0,1}(f): \mathcal{C}_{0,1} A \rightarrow \mathcal{C}_{0,1} B$ is an empty map in the case when $A=\varnothing$ and it is identity $i d_{\{\perp\}}$ otherwise.

We will now show that $\mathcal{C}_{0,1}$ does not preserve preimages. If we consider an inclusion map $i:\{0\} \rightarrow\{0,1\}$ and a subset $\{1\} \subseteq\{0,1\}$, then for the preimage $i^{-1}(\{1\})=\varnothing$ we have

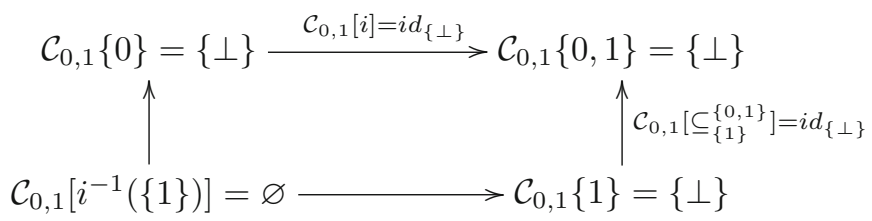

The functor $\mathcal{C}_{0,1}$ does not preserve preimages in the case when they are empty. At the same time, for any class $\mathrm{K}$ of $\mathcal{C}_{0,1}$-coalgebras we have $\mathcal{H} \mathcal{S}(\mathrm{K})=\mathcal{S H}(\mathrm{K})$. That is why the notion that should be investigated to be equivalent to $\mathcal{H} \mathcal{S}=$ $\mathcal{S H}$ is the notion of preservation of non-empty preimages.

We say that a natural transformation $\mu: G \rightarrow T$ from a functor $G$ to a functor $T$ is injective whenever for any set $A$ the mapping $\mu_{A}: G A \rightarrow T A$ is an injective mapping. We say that a functor $G$ is a subfunctor of a functor $T$ whenever there exists an injective natural transformation from $G$ to $T$. We denote this fact by $G \leq T$. We say that $\vec{a} \in T A$ is a distinguished point (see [7]) if there is a natural transformation $\mu: \mathcal{C}_{0,1} \rightarrow T$ such that $\mu(\perp)=\vec{a}$. An element $\vec{a} \in T A$ is undistinguished if it is not a distinguished point. Note that by the definition of $\mathcal{C}_{0,1}$ any natural transformation $\mu: \mathcal{C}_{0,1} \rightarrow T$ is injective. Thus, $\mathcal{C}_{0,1} \leq T$ if and only if for a set $A$ there is a distinguished point $\vec{a} \in T A$.

Definition 2.1 ([1]). A distinguished point $\mu_{X}(\perp) \in T X$, where $\mu: \mathcal{C}_{0,1} \rightarrow T$, is called standard whenever $T \varnothing$ contains precisely one element, say $\vec{s}$, such that for every non-empty set $X$ it is sent by the $T$-image of the empty map $\epsilon_{X}: \varnothing \rightarrow X$ exactly on the image $\mu_{X}(\perp)$.

Definition 2.2 ([1]). A functor $T$ : Set $\rightarrow$ Set is called a standard functor provided that

(1) it preserves inclusions, i.e., for any inclusion map $\subseteq_{A}^{B}: A \rightarrow B$, the map $T\left(\subseteq_{A}^{B}\right)$ is an inclusion map, that is, $T\left(\subseteq_{A}^{B}\right)=\subseteq_{T A}^{T B}$,

(2) all of its distinguished points are standard.

Remark 2.3. Every functor $T$ : Set $\rightarrow$ Set preserves non-empty intersections of finitely many sets (see [7]), but a standard functor preserves also the empty intersections of finitely many sets. Hence, e.g., $\mathcal{C}_{0,1}$ is not a standard functor but $\mathcal{C}_{1}$ sending any set to $\{\perp\}$ is standard.

Theorem 2.4 ([1, p. 132]). For each Set-endofunctor $T$ there exists a standard Set-endofunctor $T^{\prime}$ such that the restrictions of $T$ and $T^{\prime}$ to all non-empty sets and non-empty maps are naturally isomorphic. 
If we only investigate the preservation of non-empty preimages, then by Theorem 2.4 we may restrict ourselves to standard functors. Therefore, throughout this paper without loss of generality we will assume that a functor $T$ we deal with is standard.

We will now translate the notion of a functor that does not preserve nonempty preimages to the language of standard functors. A functor $T$ : Set $\rightarrow$ Set does not preserve non-empty preimages whenever there is a mapping $f: A \rightarrow B$, a subset $C \subset B$ such that $f^{-1}(C) \neq \varnothing$ and an element $\vec{a} \in T A$ such that $T f(\vec{a}) \in T C$ and $\vec{a} \notin T\left[f^{-1}(C)\right]$.

The notion of a subcoalgebra may also be stated more easily in the language of standard functors. Namely, suppose $\mathbb{X}=\langle X, \xi\rangle$ is a $T$-coalgebra and let $S \subseteq X$. There is a structure $\sigma: S \rightarrow T S$ turning $\langle S, \sigma\rangle$ into a subcoalgebra of $\mathbb{X}$ if and only if $\xi(S) \subseteq T S$ (see [2] for details). The structure $\sigma: S \rightarrow T S$ is defined by $\sigma:=\xi_{\mid S}$. Therefore, subcoalgebras of a given coalgebra $\mathbb{X}$ can be understood as subsets of $X$ 'closed' under the structure map $\xi$.

We have the following.

Lemma 2.5. Let $T:$ Set $\rightarrow$ Set be a functor and let $f: A \rightarrow B$ be a mapping, and $S \subseteq A$. Then

$$
[T f](T S)=T[f(S)]
$$

Proof. It is clear that $[T f](T S) \subseteq T[f(S)]$. To see that the equality between the two sets holds, notice that the restriction $f_{\mid S}: S \rightarrow f(S)$ of the mapping $f$ is onto and therefore an epimorphism. Any Set-endofunctor preserves epis and hence $T\left(f_{\mid S}\right): T S \rightarrow T[f(S)]$ is also onto. That is why $\left[T f_{\mid S}\right](T S)=$ $[T f](T S)=T[f(S)]$.

A functor $T$ is said to be connected if $|T 1|=1$. Let $\mathcal{I} d$ denote the identity functor.

Lemma 2.6 ([10]). Let $T$ : Set $\rightarrow$ Set be a standard connected functor. Then either $T$ contains exactly one isomorphic copy of $\mathcal{I} d$ as a subfunctor or it contains exactly one isomorphic copy of $\mathcal{C}_{0,1}$ as a subfunctor.

If $T$ is a connected functor and if $\mathcal{C}_{0,1} \leq T$, then by Lemma 2.6 we know that the set $T A$ contains precisely one distinguished point. Therefore, whenever we speak of a connected functor with $\mathcal{C}_{0,1} \leq T$, we denote the distinguished element of $T A$ by $\perp$. Moreover, if $T$ is a connected functor with $\mathcal{I} d \leq T$, then by Lemma 2.6 the functor $\mathcal{C}_{0,1}$ is not a subfunctor of $T$. This means that all elements from $T X$ are undistinguished.

Lemma 2.7 ([8, Prop. II.4]). Let $T:$ Set $\rightarrow$ Set be a functor and let $\vec{a} \in T A$. The following conditions are equivalent:

- there are two non-empty disjoint subsets $U_{1}, U_{2} \subset A$ with $\vec{a} \in T U_{1}$ and $\vec{a} \in T U_{2}$

- the element $\vec{a}$ is a distinguished point. 
Let $T$ be an arbitrary Set-endofunctor and $A$ be a set. For any $\vec{a} \in T A$ define a collection $\mathrm{Flt}_{A}(\vec{a})$ of subsets of $A$ as follows:

$$
\mathrm{Flt}_{A}(\vec{a}):= \begin{cases}\{U \subseteq A \mid \vec{a} \in T U\} & \text { if } \vec{a} \text { is not distinguished, } \\ \mathcal{P}(A) & \text { otherwise. }\end{cases}
$$

It has been shown in [8] that for an undistinguished point $\vec{a} \in T A$ the collection $\mathrm{Flt}_{A}(\vec{a})$ forms a filter on $A$. By Lemma 2.7 it follows that $\varnothing \in \mathrm{Flt}_{A}(\vec{a})$ if and only if $\vec{a}$ is a distinguished point.

Let $\mathcal{F}$ denote the functor which assigns to every set $A$ the set

$$
\{\mathcal{U} \mid \mathcal{U} \text { is a filter on } A\} \cup\{\mathcal{P}(A)\}
$$

and to every mapping $f: A \rightarrow B$ the mapping

$$
\mathcal{F}(f): \mathcal{F} A \rightarrow \mathcal{F} B ; \quad \mathcal{G} \mapsto\{V \subseteq B \mid f(W) \subseteq V \text { for some } W \in \mathcal{G}\} .
$$

For any set $A$ we may now treat Flt $_{A}$ as a mapping

$$
\mathrm{Flt}_{A}: T A \rightarrow \mathcal{F} A .
$$

The collection Flt $=\left\{\mathrm{Flt}_{A}\right\}_{A \in \text { Set }}$ is a transformation from $T$ to $\mathcal{F}$. It is worth noting that Flt need not be a natural transformation from $T$ to $\mathcal{F}$. We have the following two theorems.

Theorem 2.8 ([8]). For any injective mapping $f: A \rightarrow B$ and any $\vec{a} \in T A$, we have

$$
\mathrm{Flt}_{B}(T f(\vec{a}))=[\mathcal{F} f]\left(\mathrm{Flt}_{A}(\vec{a})\right) .
$$

Theorem 2.9 ([8, Prop. VII.5]). The transformation Flt: $T \rightarrow \mathcal{F}$ is a natural transformation, i.e., for any $\vec{a} \in T A$ and any mapping $f: A \rightarrow B$ we have

$$
\mathrm{Flt}_{B}(T f(\vec{a}))=[\mathcal{F} f]\left(\mathrm{Flt}_{A}(\vec{a})\right),
$$

if and only if $T$ preserves non-empty preimages.

\section{Functors not preserving non-empty preimages on undistinguished points}

Definition 3.1. We say that a Set-endofunctor $T$ does not preserve nonempty preimages on undistinguished points whenever there exist a mapping $f: A \rightarrow B$, a subset $C \subset B$ with $f^{-1}(C) \neq \varnothing$ and an element $\vec{a} \in T A$ such that $T f(\vec{a})$ is an undistinguished point, $T f(\vec{a}) \in T C$ and $\vec{a} \notin T\left[f^{-1}(C)\right]$.

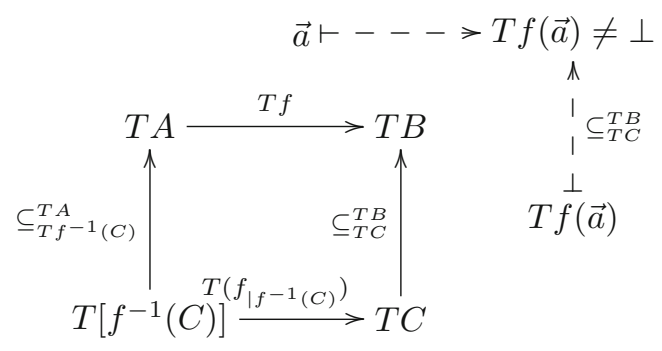


Note that the element $\vec{a}$ in the above definition cannot be a distinguished point. Because if $\vec{a} \in T A$ was distinguished, then for any mapping $f: A \rightarrow B$ the element $T f(\vec{a})$ would also be a distinguished point.

We stress that the only difference between the notion of a functor not preserving non-empty preimages and a functor not preserving non-empty preimages on undistinguished points is the additional assumption in the formulation of the latter which says that the element $\operatorname{Tf}(\vec{a}) \neq \perp$.

The following lemma is a consequence of Lemma 2.6.

Lemma 3.2. If $T$ : Set $\rightarrow$ Set is a connected functor with $\mathcal{I} d \leq T$ which does not preserve non-empty preimages, then $T$ does not preserve non-empty preimages on undistinguished points.

Proof. By Lemma 2.6 we know there is no natural transformation $\mu: \mathcal{C}_{0,1} \rightarrow T$. This means that for any set $B$ the set $T B$ does not contain distinguished points. Hence, for any set $A$, any element $\vec{a} \in T A$ and a mapping $f: A \rightarrow B$, the element $\operatorname{Tf}(\vec{a}) \in T B$ is undistinguished.

The example below shows that there are connected functors with $\mathcal{C}_{0,1} \leq T$ that do not preserve non-empty preimages on undistinguished points.

Example 3.3. Let $G$ be the functor $\operatorname{Hom}(4,-)$, i.e., $G A=\left\{\left(a_{0}, a_{1}, a_{2}, a_{3}\right) \mid\right.$ $\left.a_{i} \in A\right\}$ for any set $A$, and let $\nu: G \rightarrow T$ be an epitransformation given by the following equations:

$$
\begin{array}{cl}
\nu_{Y}(y, y, y, y)=\nu_{Y}(z, z, z, z), & \text { for any } Y \text { and for any } y, z \in Y, \\
\nu_{Y}\left(w_{1}, y, y, z\right)=\nu_{Y}\left(w_{2}, y, y, z\right), & \text { for any } Y \text { and for any } w_{1}, w_{2}, y, z \in Y .
\end{array}
$$

For $(v, w, y, z) \in Y^{4}$ let us introduce the following notation:

$$
[v, w, y, z]_{\nu}^{Y}:=\left\{\left(v^{\prime}, w^{\prime}, y^{\prime}, z^{\prime}\right) \in Y^{4} \mid \nu_{Y}(v, w, y, z)=\nu_{Y}\left(v^{\prime}, w^{\prime}, y^{\prime}, z^{\prime}\right)\right\} .
$$

The functor $T$ is connected and contains a copy of $\mathcal{C}_{0,1}$ as a subfunctor. We will show that $T$ does not preserve non-empty preimages of undistinguished points: let $A=\{a, b, c, d\}$ be a 4-element set, let $B=\{a, c, d\}, C=\{c, d\}$ and let $f: A \rightarrow B$ be a mapping sending $b$ to $c$ and being an identity on $a, c, d$. Then $T$ does not preserve the preimage of $[c, c, c, d]_{\nu}^{C}$. Indeed, the inclusion $i: C \rightarrow B$ sends $[c, c, c, d]_{\nu}^{C}$ to $[c, c, c, d]_{\nu}^{B}=[a, c, c, d]_{\nu}^{B}$ and $T(f)$ sends $[a, b, c, d]_{\nu}^{A}$ to $[a, c, c, d]_{\nu}^{B} \in T B$. But $f^{-1}(C)=\{b, c, d\}$ and $[a, b, c, d]_{\nu}^{A}$ cannot be an image of any element from $T f^{-1}(C)=T\{b, c, d\}$.

Throughout this section we will assume that $T:$ Set $\rightarrow$ Set is a standard connected functor not preserving non-empty preimages on undistinguished points. Our aim is to find a $T$-coalgebra $\mathbb{X}=\langle X, \xi\rangle$ for which $\mathcal{H} \mathcal{S}(\mathbb{X}) \neq \mathcal{S H}(\mathbb{X})$. In order to construct such a coalgebra we need to introduce some ingredients and show some of their properties.

Take sets $A, B$ and $C$, a mapping $f: A \rightarrow B$ and an element $\vec{a} \in T A$ as in Definition 3.1. Therefore, $T f(\vec{a}) \in T C, \vec{a} \in T A \backslash T\left[f^{-1}(C)\right]$ and $T f(\vec{a}) \neq \perp$. We have the following lemmata. 
Lemma 3.4. The following is true:

$$
\vec{a} \notin T\left[A \backslash f^{-1}(C)\right] .
$$

Proof. If $\vec{a} \in T\left[A \backslash f^{-1}(C)\right]$, then by Lemma 2.5, Tf( $\left.\vec{a}\right) \in[T f]\left(A \backslash f^{-1}(C)\right)$. Because $T f(\vec{a}) \in[T f]\left(A \backslash f^{-1}(C)\right) \cap T C$ and because $f\left[A \backslash f^{-1}(C)\right] \cap C=\varnothing$ by [7] we imply that $T f(\vec{a})$ has to be a distinguished point. This contradicts the assumptions.

Lemma 3.5. We have

$$
f(A) \cap C \neq \varnothing .
$$

Proof. Assume that $f(A) \cap C=\varnothing$. By [7] the set $T f(A) \cap T C$ contains only the distinguished point. This is a contradiction since $\operatorname{Tf}(\vec{a}) \in T f(A) \cap T C$ and $\operatorname{Tf}(\vec{a}) \neq \perp$.

By Lemma 3.5 and by the fact that any Set-endofunctor preserves nonempty intersections (see [7]) we may assume without a loss of generality that $f: A \rightarrow B$ is onto. Indeed, if $f: A \rightarrow B$ is not a surjection, then the map $f$ may be replaced with $f^{\prime}: A \rightarrow f(A)$ and the set $C$ with $C^{\prime}=f(A) \cap C$.

We will denote the only element of the set $T\{v\}$ by $*_{v}$, i.e., $T\{v\}=\left\{*_{v}\right\}$. Note that if $\mathcal{C}_{0,1} \leq T$, then $*_{v}=\perp$.

Define the set $X:=A+A=A \times\{1\} \cup A \times\{2\}$. Let $\vec{a}_{1} \in T X$ be defined as $\vec{a}_{1}:=T i_{1}(\vec{a})$, where $i_{1}: A \rightarrow A \times\{1\} \cup A \times\{2\} ; a \mapsto(a, 1)$. Similarly, we define $\vec{a}_{2} \in T X$. That is, we take $\vec{a}_{2}=T i_{2}(\vec{a})$, where $i_{2}: A \rightarrow A \times\{1\} \cup A \times\{2\} ; a \mapsto$ $(a, 2)$.

Take the set $X$ to be the carrier of our coalgebra $\mathbb{X}$ and let the structure $\xi: X \rightarrow T X$ of the coalgebra $\mathbb{X}$ be defined as

$$
\xi(v)= \begin{cases}\vec{a}_{2} & \text { for } v \in A \times\{1\}, \\ \vec{a}_{1} & \text { for } v \in f^{-1}(C) \times\{2\}, \\ *_{v} & \text { for } v \in\left[A \backslash f^{-1}(C)\right] \times\{2\} .\end{cases}
$$

We are now going to list some basic facts about subcoalgebras of the coalgebra $\mathbb{X}$.

Lemma 3.6. If $\mathbb{S} \leq \mathbb{X}$ with $S \cap A \times\{1\} \neq \varnothing$, then $S \cap f^{-1}(C) \times\{2\} \neq \varnothing$ and $S \cap\left[A \backslash f^{-1}(C)\right] \times\{2\} \neq \varnothing$. If $S \cap f^{-1}(C) \times\{2\} \neq \varnothing$, then $S \cap A \times\{1\} \neq \varnothing$.

Proof. This follows directly from the definition of $\vec{a}_{1}, \vec{a}_{2}$ and the fact that $\vec{a} \in T A, \vec{a} \notin T\left[f^{-1}(C)\right]$ and $\vec{a} \notin T\left[A \backslash f^{-1}(C)\right]$.

Lemma 3.7. For any non-empty subcoalgebra $\mathbb{S} \leq \mathbb{X}$ we have

$$
S \cap\left[A \backslash f^{-1}(C) \times\{2\}\right] \neq \varnothing .
$$

Proof. Indeed, if $v \in S$ and $v \in f^{-1}(C) \times\{2\}$, then by Lemma 3.6 we have $S \cap[A \times\{1\}] \neq \varnothing$. Now, if $v \in S \cap[A \times\{1\}]$, then again by Lemma 3.6 it implies that $S \cap\left[A \backslash f^{-1}(C) \times\{2\}\right] \neq \varnothing$. 
Notice that each element from the subset $\left[A \backslash f^{-1}(C)\right] \times\{2\}$ forms a oneelement subcoalgebra of $\mathbb{X}$. In other words, Lemma 3.7 states that each nonempty subcoalgebra of $\mathbb{X}$ contains a one-element subcoalgebra.

Let $Y=A+B=A \times\{1\} \cup B \times\{2\}$. Firstly, define $h: X \rightarrow Y$ as

$$
h(v)= \begin{cases}v & \text { for } v \in A \times\{1\}, \\ (f(a), 2) & \text { for } v=(a, 2) \in A \times\{2\} .\end{cases}
$$

Now, we are ready to define the structure $\psi: Y \rightarrow T Y$ as

$$
\psi(v)= \begin{cases}T h\left(\vec{a}_{2}\right) & \text { for } v \in A \times\{1\}, \\ \vec{a}_{1} & \text { for } v \in C \times\{2\}, \\ *_{v} & \text { for } v \in[B \backslash C] \times\{2\} .\end{cases}
$$

It is not hard to prove that $h: X \rightarrow Y$ is a homomorphism between $\mathbb{X}$ and $\mathbb{Y}=\langle Y, \psi\rangle$. Moreover, the following properties of $\mathbb{Y}$ hold.

Lemma 3.8. For any subcoalgebra $\mathbb{S} \leq \mathbb{Y}$ the condition $A \times\{1\} \cap S \neq \varnothing$ is equivalent to $C \times\{2\} \cap S \neq \varnothing$. Moreover, $\psi(A+C) \subseteq T[A+C]$.

Proof. This follows directly from the definition of $\mathbb{Y}$ and the properties of the element $T h\left(\vec{a}_{2}\right)$, which is isomorphic to the element $T f(\vec{a})$.

By Lemma 3.8 it follows that $\left\langle A+C, \psi_{\mid A+C}\right\rangle$ forms a subcoalgebra of $\mathbb{Y}$. We will now show the following theorem.

Theorem 3.9. The coalgebra $\left\langle A+C, \psi_{\mid A+C}\right\rangle$ belongs to the class $\mathcal{S H}(\mathbb{X})$ but it does not belong to the class $\mathcal{H} \mathcal{S}(\mathbb{X})$.

Proof. It is clear that $\left\langle A+C, \psi_{\mid A+C}\right\rangle \in \mathcal{S H}(\mathbb{X})$. To show that the coalgebra $\left\langle A+C, \psi_{\mid A+C}\right\rangle \notin \mathcal{H} \mathcal{S}(\mathbb{X})$ observe that by Lemma 3.7 any non-empty subcoalgebra $\mathbb{S}$ of $\mathbb{X}$ contains some elements from the subset $\left[A \backslash f^{-1}(C)\right] \times\{2\}$. That is, for each subcoalgebra $\mathbb{S} \leq \mathbb{X}$ there is $v \in S$ such that $\left\langle\{v\}, \xi_{\mid\{v\}}\right\rangle$ forms a one-element subcoalgebra of $\mathbb{S}$. Assume by contradiction that $\left\langle A+C, \psi_{\mid A+C}\right\rangle \in$ $\mathcal{H}(\mathbb{X})$. This means that there is $\mathbb{S} \leq \mathbb{X}$ whose homomorphic image is the coalgebra $\left\langle A+C, \psi_{\mid A+C}\right\rangle$. This would mean that the image of the element $v \in S$ forms a one-element subcoalgebra of $\left\langle A+C, \psi_{\mid A+C}\right\rangle$. This is impossible, since by Lemma 3.8 the coalgebra $\left\langle A+C, \psi_{\mid A+C}\right\rangle$ does not contain any one-element subcoalgebras. A contradiction.

\section{Functors preserving non-empty preimages on undistinguished points}

In this section we will present a general setting which allows us to construct many examples of coalgebras for functors that fail to satisfy $\mathcal{S H}=\mathcal{H} \mathcal{S}$. At the time of writing this paper we were unable to find a complete solution to the $\mathcal{H} \mathcal{S}=\mathcal{S H}$ problem. 
4.1. Standing Hypothesis. In the rest of the paper we assume that the functor $T$ : Set $\rightarrow$ Set is connected, standard, it does not preserve non-empty preimages, it is not the case that $T$ does not preserve non-empty preimages on undistinguished points and it has a distinguished point, say $\mu_{X}(\perp) \in T X$ for a natural transformation $\mu: \mathcal{C}_{0,1} \rightarrow T$ and a non-empty set $X$. For any non-empty set $X$, we will denote the unique element $\mu_{X}(\perp)$ by $\perp_{X}$ or simply $\perp$. We know that if $T$ is a connected Set-endofunctor with $\mathcal{C}_{0,1} \leq T$, then $T$ preserves non-empty preimages if and only if the restrictions of $T$ and $\mathcal{C}_{0,1}$ to non-empty sets are naturally equivalent (see [8] for a proof). Therefore, $T$ is distinct from a constant functor.

We have the following lemma.

Lemma 4.1. Let $T$ be as above. Let $f: A \rightarrow B$ be a mapping and $\vec{a} \in T A$ such that $[T f](\vec{a}) \neq \perp$. Then

$$
\mathrm{Flt}_{B}([T f](\vec{a}))=[\mathcal{F} f]\left(\mathrm{Flt}_{A}(\vec{a})\right) .
$$

Proof. It is always the case that $[\mathcal{F} f]\left(\mathrm{Flt}_{A}(\vec{a})\right) \subseteq \mathrm{Flt}_{B}([T f](\vec{a}))$ (see $\left.[3]\right)$. By contradiction assume that $[\mathcal{F} f]\left(\mathrm{Flt}_{A}(\vec{a})\right) \subset \mathrm{Flt}_{B}([T f](\vec{a}))$. In general we have

$$
\begin{aligned}
& {[\mathcal{F} f]\left(\mathrm{Flt}_{A}(\vec{a})\right)=\left\{W \subseteq B \mid f(U) \subseteq W \text { for } U \in \mathrm{Flt}_{A}(\vec{a})\right\}} \\
& \quad=\{W \subseteq B \mid f(U) \subseteq W \text { for } \vec{a} \in T U\}=\left\{V \subseteq B \mid \vec{a} \in T\left[f^{-1}(V)\right]\right\}
\end{aligned}
$$

and

$$
\mathrm{Flt}_{B}([T f](\vec{a}))=\{V \subseteq B \mid[T f](\vec{a}) \in T V\} .
$$

We see that if there was a set $C \subseteq B$ such that $C \notin[\mathcal{F} f]\left(\mathrm{Flt}_{A}(\vec{a})\right)$ and $C \in \operatorname{Flt}_{B}([T f](\vec{a}))$, then $\vec{a} \notin T\left[f^{-1}(C)\right]$ and $[T f](\vec{a}) \in T C$. Since $[T f](\vec{a}) \neq \perp$ we get that $T$ does not preserve non-empty preimages on undistinguished points. A contradiction.

We define

$$
\lambda:=\text { a cardinal number for which }|T \lambda|>1,
$$

and assume that $A$ is a set with $|A|=\lambda$ and $\vec{a} \in T A$ is an undistinguished point.

In the general setting we only assume that we have a $T$-coalgebra $\mathbb{B}=\langle B, \beta\rangle$ and two undistinguished elements $\vec{a}_{B, 1} \in T B$ and $\vec{a}_{B, 2} \in T B$ satisfying the following properties.

(1) $\perp \notin \beta(B)$ and $\vec{a}_{B, 1}, \vec{a}_{B, 2} \notin \beta(B)$.

(2) If there are $\mathbb{S} \leq\langle B, \beta\rangle \leq\langle C, \gamma\rangle$ with

- $\vec{a}_{B, 1}, \vec{a}_{B, 2} \in T S$,

- $\gamma(C \backslash B)=\left\{\vec{a}_{B, 1}, \perp\right\}$,

then given a homomorphism $h: \mathbb{S} \rightarrow \mathbb{C}$ such that $T h\left(\vec{a}_{B, 1}\right)=\vec{a}_{B, 1} \in$ $T B \subseteq T C$ we have $T h\left(\vec{a}_{B, 2}\right) \notin \beta(B) \cup\left\{\vec{a}_{B, 1}\right\} \cup\{\perp\}$.

To see some classes of functors for which there is a coalgebra and elements satisfying conditions (1) and (2), the reader is referred to Subsections 4.2-4.5. 
We will now show that given such a $T$-coalgebra $\mathbb{B}$ and elements $\vec{a}_{B, 1}, \vec{a}_{B, 2}$ satisfying (1), (2) we are able to construct a $T$-coalgebra $\mathbb{X}$ such that $\mathcal{S H}(\mathbb{X}) \neq$ $\mathcal{H S}(\mathbb{X})$.

Assume that the set $A$ is disjoint from $B, 2^{B}$ and $2^{2^{B}}$, and let

$$
X=B \cup A \cup 2^{B} \cup 2^{2^{B}} .
$$

Finally, recall that we have $\vec{a} \in T A \subset T X$. We will define the structure $\xi: X \rightarrow T X$ as follows:

$$
\xi(v)= \begin{cases}\beta(v) & \text { for } v \in B, \\ \vec{a}_{B, 2} & \text { for } v \in A, \\ \vec{a} & \text { for } v \in 2^{B}, \\ \vec{a}_{B, 1} & \text { for } v \in 2^{2^{B}} .\end{cases}
$$

Now let $Y=B \cup\{s\} \cup 2^{B} \cup 2^{2^{B}}$ and let $\psi: Y \rightarrow T Y$ be defined as follows:

$$
\psi(v)= \begin{cases}\beta(v) & \text { for } v \in B, \\ \vec{a}_{B, 2} & \text { for } v=s, \\ \perp & \text { for } v \in 2^{B} \\ \vec{a}_{B, 1} & \text { for } v \in 2^{2^{B}} .\end{cases}
$$

It is clear that $\mathbb{Y}=\langle Y, \psi\rangle$ is a homomorphic image of $\mathbb{X}$. Moreover, the subset $B \cup 2^{B} \cup 2^{2^{B}}$ is closed under the structure map $\psi$ of the coalgebra $\mathbb{Y}$. In other words, $\left\langle B \cup 2^{B} \cup 2^{2^{B}}, \psi_{\mid}\right\rangle \leq \mathbb{Y}$ and that is why $\left\langle B \cup 2^{B} \cup 2^{2^{B}}, \psi_{\mid}\right\rangle \in \mathcal{S H}(\mathbb{X})$. We are now going to show that $\left\langle B \cup 2^{B} \cup 2^{2^{B}}, \psi_{\mid}\right\rangle \notin \mathcal{H} \mathcal{S}(\mathbb{X})$. Assume by contradiction that there is a subcoalgebra $\mathbb{S}=\left\langle S, \xi_{\mid}\right\rangle \leq \mathbb{X}$ whose homomorphic image under a homomorphism $h: S \rightarrow B \cup 2^{B} \cup 2^{2^{B}}$ is the coalgebra $\left\langle B \cup 2^{B} \cup 2^{2^{B}}, \psi_{\mid}\right\rangle$. We will derive some properties of $h$ that will lead us to a contradiction.

First of all observe that $|A| \leq|B|<\left|2^{B}\right|<\left|2^{2^{B}}\right|$. Because of cardinality issues and because $h$ is onto, the subcoalgebra $\mathbb{S}$ has to contain some elements from $2^{2^{B}}$ which are mapped to $2^{2^{B}}$ by $h$. Hence, there is an element $c \in S \cap 2^{2^{B}}$ such that $h(c) \in 2^{2^{B}} \subset Y$. Since $h$ is a homomorphism we have $T h(\xi(c))=$ $T h\left(\vec{a}_{B, 1}\right)=\psi(h(c))=\vec{a}_{B, 1}$. Therefore, for any $v \in S \cap 2^{2^{B}}$ the following is true: $\psi(h(v))=T h(\xi(v))=T h(\xi(c))=\vec{a}_{B, 1}$. Hence for any $v \in S \cap 2^{2^{B}}$ it has to be the case that $h(v) \in 2^{2^{B}}$ because otherwise $\psi(h(v))=T h(\xi(v))$ would not be equal to $\vec{a}_{B, 1}$. Similarly, by cardinality issues $2^{B} \cap h\left(S \cap 2^{B}\right) \neq \varnothing$. Using an argument similar to the one above we show that $h\left(S \cap 2^{B}\right) \subseteq 2^{B}$. To sum up:

$$
\begin{gathered}
S \cap 2^{2^{B}} \neq \varnothing \text { with } h\left(S \cap 2^{2^{B}}\right) \subseteq 2^{2^{B}}, \\
S \cap 2^{B} \neq \varnothing \text { with } h\left(S \cap 2^{B}\right) \subseteq 2^{B} .
\end{gathered}
$$

From the construction of $\mathbb{X}$ it follows that $\mathbb{S}$ also contains some elements from the subset $A \subset X$ and some elements from $B$ since $\vec{a} \in T S$ and $\vec{a}_{B, 1} \in T S$. 
Moreover, because $S \cap A \neq \varnothing$ we also have $\vec{a}_{B, 2} \in T S$. That is,

$$
S \cap A \neq \varnothing, \quad S \cap B \neq \varnothing \quad \text { and } \quad \mathbb{S} \cap \mathbb{B} \leq \mathbb{B}<\mathbb{X} .
$$

For any $v \in S \cap 2^{2^{B}}$ we have $h(v) \in 2^{2^{B}}$ and therefore, the following equality is true:

$$
T h(\xi(v))=T h\left(\vec{a}_{B, 1}\right)=\psi(h(v))=\vec{a}_{B, 1} \in T B \subset T\left[B \cup 2^{B} \cup 2^{2^{B}}\right] .
$$

Therefore, if we consider the coalgebras $\mathbb{S} \cap \mathbb{B} \leq \mathbb{B}<\left\langle B \cup 2^{B} \cup 2^{2^{B}}, \psi_{\mid}\right\rangle$, a homomorphism $h_{\mid S \cap B}: \mathbb{S} \cap \mathbb{B} \rightarrow\left\langle B \cup 2^{B} \cup 2^{2^{B}}, \psi_{\mid}\right\rangle$which is a restriction of $h$, then it is true that $\operatorname{Th}\left(\vec{a}_{B, 1}\right)=T h_{S \cap B}\left(\vec{a}_{B, 1}\right)=\vec{a}_{B, 1}$. This means that we may apply property (2) and get

$$
T h[\xi(S \cap B)]=T h_{\mid S \cap B}[\xi(S \cap B)]=T h_{\mid S \cap B}[\beta(S \cap B)] \subseteq \beta(B) .
$$

By (2) we also see that $T h_{\mid S \cap B}\left(\vec{a}_{B, 2}\right)=T h\left(\vec{a}_{B, 2}\right) \notin \beta(B) \cup\left\{\vec{a}_{B, 1}, \perp\right\}$. This is a contradiction, because for any $v \in S \cap A$ the following is true:

$$
T h\left(\vec{a}_{B, 2}\right)=T h(\xi(v))=\psi(h(v)) \in \psi\left(B \cup 2^{B} \cup 2^{2^{B}}\right)=\beta(B) \cup\left\{\vec{a}_{B, 1}, \perp\right\} .
$$

Theorem 4.2. Let $T:$ Set $\rightarrow$ Set be a connected functor that preserves nonempty preimages on undistinguished points and $\mathcal{C}_{0,1} \leq T$. Let $\lambda$ be any cardinal number for which $|T \lambda|>1$. Moreover, assume that there is a T-coalgebra $\mathbb{B}$ with $|B| \geq \lambda$ and elements $\vec{a}_{B, 1}, \vec{a}_{B, 2} \in T B$ with properties (1) and (2) listed above. Then there is a $T$-coalgebra $\mathbb{X}$ for which $\mathcal{S H}(\mathbb{X}) \neq \mathcal{H} \mathcal{S}(\mathbb{X})$.

4.2. Non-free filters. Let $T, \perp, A, \vec{a} \in T A, \lambda$ be as in the Standing Hypothesis. In this subsection we will present a construction of a $T$-coalgebra $\langle B, \beta\rangle$ and elements $\vec{a}_{B, 1}, \vec{a}_{B, 2} \in T B$ for a functor $T$ satisfying an additional property: the intersection $\bigcap \mathrm{Flt}_{A}(\vec{a})$ is non-empty, i.e., the filter $\mathrm{Flt}_{A}(\vec{a})$ is not free.

We know that any finitary functor different from a constant functor has elements whose filters are not free. That is why the construction presented in this subsection works for finitary connected functors preserving non-empty preimages on undistinguished points and having $\mathcal{C}_{0,1}$ as a subfunctor.

Define

$$
B:=[A+A] \cup\{x, y\}=[A \times\{1\}] \cup[A \times\{2\}] \cup\{x, y\} .
$$

Let $z \in \bigcap \mathrm{Flt}_{A}(\vec{a})$. Define the mapping $i_{x}^{k}: A \rightarrow B$ for $k=1,2$ as follows:

$$
\begin{aligned}
i_{x}^{k}(v) & :=(v, k) \text { for } v \neq z, \text { and } \\
i_{x}^{k}(z) & :=x .
\end{aligned}
$$

Similarly, we define $i_{y}^{k}: A \rightarrow B$ for $k=1,2$ :

$$
\begin{aligned}
i_{y}^{k}(v) & :=(v, k) \text { for } v \neq z, \text { and } \\
i_{y}^{k}(z) & :=y
\end{aligned}
$$


Moreover, let $i_{1}: A \rightarrow B ; a \mapsto(a, 1)$ and $i_{2}: A \rightarrow B ; a \mapsto(a, 2)$. We introduce the following elements:

$$
\begin{aligned}
\vec{a}_{x}^{k} & :=T i_{x}^{k}(\vec{a}), \\
\vec{a}_{y}^{k} & :=T i_{y}^{k}(\vec{a}), \\
\vec{a}_{1} & :=T i_{1}(\vec{a}), \\
\vec{a}_{2} & :=T i_{2}(\vec{a}) .
\end{aligned}
$$

It is clear that for $k=1,2$ we have

$$
x \in \bigcap \operatorname{Flt}_{B}\left[T i_{x}^{k}(\vec{a})\right] \text { and } y \in \bigcap \operatorname{Flt}_{B}\left[T i_{y}^{k}(\vec{a})\right] .
$$

We will construct a coalgebra on the carrier $B$. We define the structure $\beta: B \rightarrow T B$ as follows:

$$
\beta(v)= \begin{cases}\vec{a}_{y}^{1} & \text { for } v=x, \\ \vec{a}_{x}^{2} & \text { for } v=y, \\ \vec{a}_{1} & \text { for } v \in A \times\{1\}, \\ \vec{a}_{2} & \text { for } v \in A \times\{2\} .\end{cases}
$$

Now let $\vec{a}_{B, 1}=\vec{a}_{x}^{1}$ and $\vec{a}_{B, 2}=\vec{a}_{y}^{2}$. First of all observe that property (1) from the Standing Hypothesis is satisfied. To show that (2) holds take any subcoalgebra $\mathbb{S} \leq \mathbb{B}$ with $\vec{a}_{B, 1} \in T S$ and $\vec{a}_{B, 2} \in T S$. Also consider a coalgebra $\mathbb{C}$ with $\mathbb{B} \leq \mathbb{C}, \gamma(C \backslash B)=\left\{\vec{a}_{B, 1}, \perp\right\}$ and a homomorphism $h: \mathbb{S} \rightarrow \mathbb{C}$ such that $T h\left(\vec{a}_{B, 1}\right)=\vec{a}_{B, 1}$. The fact $\vec{a}_{B, 1}=\vec{a}_{x}^{1} \in T S$ and $\vec{a}_{B, 2}=\vec{a}_{y}^{2} \in T S$ implies that $x, y \in S$ and

$$
S \cap A \times\{1\} \neq \varnothing \neq S \cap A \times\{2\}
$$

\section{Lemma 4.3.}

$$
\begin{aligned}
h(S \cap A \times\{1\}) & \subseteq S \cap A \times\{1\}, \\
h(S \cap A \times\{2\}) & \subseteq S \cap A \times\{2\}, \\
h(x) & =x, \\
h(y) & =y .
\end{aligned}
$$

Proof. If $T h\left(\vec{a}_{x}^{1}\right)=\operatorname{Th}\left(\vec{a}_{B, 1}\right)=\vec{a}_{B, 1}=\vec{a}_{x}^{1}$, then by Lemma 4.1 we have

$$
\mathcal{F} h\left(\mathrm{Flt}_{S}\left(\vec{a}_{x}^{1}\right)\right)=\left\{W \mid f(U) \subseteq W \text { and } U \in \mathrm{Flt}_{S}\left(\vec{a}_{x}^{1}\right)\right\}=\operatorname{Flt}_{C}\left(\vec{a}_{x}^{1}\right) .
$$

Therefore, there is a set $U \subseteq S \cap[A \times\{1\}] \cup\{x\}$ such that $h(U) \subseteq[A \times\{1\}] \cup\{x\}$ and there is a point $v \in S \cap[A \times\{1\}] \cup\{x\}$ which satisfies $h(v)=x$. By the fact $\beta(A \times\{1\})=\left\{\vec{a}_{1}\right\} \neq\left\{\vec{a}_{x}^{1}\right\}=\beta(\{x\})$ and the fact that $\vec{a}_{x}^{1} \notin \gamma(C \backslash\{x\})$ if there was an element $v \in S \cap[A \times\{1\}]$ such that $v \neq x$ and $h(v)=x$, then $h(S \cap[A \times\{1\}])=\{x\}$ and

$$
T h\left(\vec{a}_{1}\right)=T h(\beta(v))=\beta(h(v))=\beta(x)=\vec{a}_{y}^{1} .
$$

This clearly implies that

$$
\mathcal{F} h\left(\mathrm{Flt}_{S}\left(\vec{a}_{1}\right)\right)=\mathrm{Flt}_{C}\left(\vec{a}_{y}^{1}\right)
$$


Thus, there is $V \subseteq S \cap A \times\{1\}$ such that $h(V) \subseteq A \times\{1\} \cup\{y\}$, which contradicts the fact that $h(S \cap[A \times\{1\}])=\{x\}$. Therefore, $h(S \cap[A \times\{1\}]) \subseteq A \times\{1\}$ and $h(x)=x$.

Now, if $h(x)=x$, then

$$
\vec{a}_{y}^{1}=\beta(x)=\gamma(x)=\gamma(h(x))=T h(\beta(x))=T h\left(\vec{a}_{y}^{1}\right) .
$$

Therefore, by applying a similar reasoning we get $h(S \cap[A \times\{2\}]) \subseteq A \times\{2\}$ and $h(y)=y$.

If we define a bijection $j: C \rightarrow C$ as

$$
j(v)= \begin{cases}v & \text { for } v \notin\{x, y\}, \\ x & \text { for } v=y \\ y & \text { for } v=x\end{cases}
$$

then

$$
j \circ h=h \circ j_{\mid S} \quad \text { and } \quad \vec{a}_{B, 2}=\vec{a}_{y}^{2}=T j\left(\vec{a}_{x}^{2}\right) .
$$

By the fact that $h(y)=y$ (see Lemma 4.3) and that $h: \mathbb{S} \rightarrow \mathbb{C}$ is a homomorphism we have

$$
T h\left(\vec{a}_{x}^{2}\right)=T h\left(\beta_{\mid S}(y)\right)=\gamma(h(y))=\gamma(y)=\vec{a}_{x}^{2} .
$$

Therefore,

$$
T h\left(\vec{a}_{B, 2}\right)=T h\left(\vec{a}_{y}^{2}\right)=T h\left[T j_{\mid S}\left(\vec{a}_{x}^{2}\right)\right]=T j\left[T h\left(\vec{a}_{x}^{2}\right)\right]=T j\left(\vec{a}_{x}^{2}\right)=\vec{a}_{y}^{2}=\vec{a}_{B, 2} .
$$

Hence, $T h\left(\vec{a}_{B, 2}\right) \notin \beta(B) \cup\left\{a_{B, 1}\right\} \cup\{\perp\}$.

The existence of a $T$-coalgebra $\mathbb{B}$ and elements $\vec{a}_{B, 1}$ and $\vec{a}_{B, 2}$ for which the properties (1) and (2) are true guarantees that the following theorem is true.

Theorem 4.4. Let $T:$ Set $\rightarrow$ Set and $A$ be as in the Standing Hypothesis. Moreover, let there be an undistinguished point $\vec{a} \in T A$ such that the set $\bigcap \mathrm{Flt}_{A}(\vec{a})$ is non-empty. Then there exists a $T$-coalgebra $\mathbb{X}$ such that $\mathcal{H} \mathcal{S}(\mathbb{X}) \neq$ $\mathcal{S H}(\mathbb{X})$.

4.3. Free filters on $\omega$. Let $T, \perp, A, \vec{a} \in T A, \lambda$ be as in the Standing Hypothesis. Throughout this subsection we assume that we deal with functors for which $\lambda=\omega$ and for which there is an undistinguished point $\vec{a} \in T A$ whose filter $\mathrm{Flt}_{A}(\vec{a})$ is free. We will present a construction of a $T$-coalgebra $\mathbb{B}$ and elements $\vec{a}_{B, 1}, \vec{a}_{B, 2}$ satisfying properties (1) and (2).

Let $B=\omega \times \omega$. For any $n=0,1,2, \ldots$ define a mapping $i_{n}: \omega \rightarrow B ; k \mapsto$ $(k, n)$ and an element from $T B$ as follows:

$$
\vec{a}_{n}=T i_{n}(\vec{a})
$$

It is clear from the definition that $\vec{a}_{n} \in T[\omega \times\{n\}] \subset T B$. Finally, let $i_{\omega}: \omega \rightarrow$ $X ; k \mapsto(1, k)$ and denote

$$
\vec{a}_{\omega}=T i_{\omega}(\vec{a})
$$


We see that $\vec{a}_{\omega} \in T[\{1\} \times \omega] \subset T[\omega \times \omega]=T B$ and $\vec{a}_{\omega} \notin T[\omega \times\{n\}]$ for any $n=0,1,2, \ldots$ We are ready to define the structure $\beta: B \rightarrow T B$ and the two elements as follows:

$$
\begin{aligned}
& \beta(v):=\vec{a}_{n+1} \text { for } v \in \omega \times\{n\}, \\
& \vec{a}_{B, 1}:=\vec{a}_{0}, \\
& \vec{a}_{B, 2}:=\vec{a}_{\omega} .
\end{aligned}
$$

Clearly $\mathbb{B}=\langle B, \beta\rangle, \vec{a}_{B, 1}$ and $\vec{a}_{B, 2}$ satisfy property (1).

To see that (2) is also satisfied take a subcoalgebra $\mathbb{S} \leq \mathbb{B}$ such that $\vec{a}_{B, 1}=$ $\vec{a}_{0} \in T S$ and a homomorphism $h: \mathbb{S} \rightarrow \mathbb{C}$ where $\mathbb{C}$ has $\mathbb{B}$ as its subcoalgebra and $T h\left(\vec{a}_{B, 1}\right)=\vec{a}_{B, 1} \in T B \subseteq T C$. If $\vec{a}_{0} \in T S$, then it means that $S$ contains a subset of $\omega \times\{0\} \subseteq B$. That is $S \cap \omega \times\{0\} \neq \varnothing$. But then it can be easily shown by induction that $S \cap \omega \times\{n\} \neq \varnothing$ for any $n=1,2, \ldots$ Moreover, if the following equality is true

$$
\operatorname{Th}\left(\vec{a}_{0}\right)=\operatorname{Th}\left(\vec{a}_{B, 1}\right)=\vec{a}_{B, 1}=\vec{a}_{0},
$$

then there is $V \subseteq S \cap \omega \times\{0\}$ such that $h(V) \subseteq \omega \times\{0\}$. This means that for $v \in V, T h\left(\vec{a}_{1}\right)=T h(\beta(v))=\gamma(h(v))=\vec{a}_{1}$. We may apply induction and show that $T h\left(\vec{a}_{n}\right)=\vec{a}_{n}$ for $n=2,3,4, \ldots$.

Now, in order to show that (2) is satisfied it is enough to prove that $T h\left(\vec{a}_{B, 2}\right) \notin \beta(B) \cup\left\{\vec{a}_{B, 1}\right\} \cup\{\perp\}$. We will start with showing that $T h\left(\vec{a}_{B, 2}\right) \notin$ $\beta(B) \cup\left\{\vec{a}_{B, 1}\right\}$. Assume by contradiction that $\operatorname{Th}\left(\vec{a}_{B, 2}\right)=\operatorname{Th}\left(\vec{a}_{\omega}\right) \in \beta(B) \cup$ $\left\{\vec{a}_{B, 1}\right\}=\left\{\vec{a}_{0}, \vec{a}_{1}, \vec{a}_{2}, \ldots\right\}$. This means that there is a number $n \in\{0,1,2, \ldots\}$ such that

$$
\mathcal{F} h\left(\mathrm{Flt}_{S}\left(\vec{a}_{\omega}\right)\right)=\left\{W \mid h(U) \subseteq W \text { and } U \in \mathrm{Flt}_{S}\left(\vec{a}_{\omega}\right)\right\}=\mathrm{Flt}_{B}\left(\vec{a}_{n}\right) .
$$

Therefore, there exists a subset $U \subseteq\{1\} \times \omega$ with $U \in \mathrm{Flt}_{S}\left(\vec{a}_{\omega}\right)$ such that $h(U) \subseteq \omega \times\{n\}$. Because the filter $\mathrm{Flt}_{S}\left(\vec{a}_{\omega}\right)$ is free and because $U \in \mathrm{Flt}_{S}\left(\vec{a}_{\omega}\right)$, we deduce that $U$ is infinite and $U \nsubseteq \omega \times\{n\}$ for any $n=0,1,2, \ldots$ This precisely means that $\beta(U)$ is an infinite subset of $\left\{\vec{a}_{k} \mid k=0,1,2, \ldots\right\}$ such that $T h(\beta(U))=\left\{\vec{a}_{n+1}\right\}$. This is a contradiction, since the mapping $T h$ is injective on the set $\left\{\vec{a}_{k} \mid k=0,1,2, \ldots\right\}$. Thus, $\operatorname{Th}\left(\vec{a}_{B, 2}\right) \notin \beta(B) \cup\left\{\vec{a}_{0}\right\}=$ $\left\{\vec{a}_{k} \mid k=0,1,2, \ldots\right\}$. Now, it is enough to show that $\operatorname{Th}\left(\vec{a}_{B, 2}\right) \neq \perp$. Recall that $\vec{a}_{B, 2}=\vec{a}_{\omega}$ was defined as an element of $T[\{1\} \times \omega] \subset T B$. Because of the fact that $T h$ is one-to-one on $\left\{a_{k} \mid k \in \omega\right\}$, the restriction $h_{\mid\{1\} \times \omega}:\{1\} \times \omega \rightarrow$ $C$ has to be a one-to-one mapping. This exactly means that $T h\left(\vec{a}_{B, 2}\right)=$ $T h_{\mid\{1\} \times \omega}\left(\vec{a}_{\omega}\right) \neq \perp$. Hence, $\operatorname{Th}\left(\vec{a}_{B, 2}\right) \notin \beta(B) \cup\left\{\vec{a}_{B, 1}\right\} \cup\{\perp\}$.

Theorem 4.5. Let $T$ : Set $\rightarrow$ Set and $A$ be as in the Standing Hypothesis. Moreover, let $\lambda=\omega$ and let there be an undistinguished point $\vec{a} \in T A$ for which the filter $\operatorname{Flt}_{A}(\vec{a})$ is free. Then there exists a T-coalgebra $\mathbb{X}$ such that $\mathcal{H S}(\mathbb{X}) \neq \mathcal{S H}(\mathbb{X})$.

The corollary below follows from Theorems 4.4 and 4.5 . 
Corollary 4.6. Let $T$ : Set $\rightarrow$ Set and $A$ be as in the Standing Hypothesis. Moreover, let $\lambda \leq \omega$. Then there exists a $T$-coalgebra $\mathbb{X}$ such that $\mathcal{H} \mathcal{S}(\mathbb{X}) \neq$ $\mathcal{S H}(\mathbb{X})$.

4.4. Free filters with $\lambda$-many disjoint stationary sets. Let $T, \perp, A$, $\vec{a} \in T A, \lambda$ be as in the Standing Hypothesis and $\lambda$ uncountable. Here we suppose that we deal with a functor $T$ for which there is an element $\vec{a} \in T A$ and a partition $\left\{A_{l}\right\}_{l \in \lambda}$ of the set $A$ into $\lambda$-many non-empty subsets such that each of them is $\mathrm{Flt}_{A}(\vec{a})$-stationary. Our aim is to find a $T$-coalgebra $\mathbb{B}$ and elements $\vec{a}_{B, 1}, \vec{a}_{B, 2}$ satisfying (1) and (2).

Recall that a binary relation $(W, R)$ is called rigid if the only homomorphism (in the sense of relational structures) with $(W, R)$ as a domain and codomain is the identity $i d_{W}$ (see [5, p. 63] and [9] for details). The following result about rigidity of binary relations will be extensively used in this subsection.

Theorem 4.7 ([9], see also [5]). For any cardinal $\kappa$ there is a binary rigid relation $(W, R)$ without loops such that $|W|=\kappa$ and for any $x \in W$ there is $y \in W$ such that $(x, y) \in R$.

Now take a rigid relation $(W, R)$ from Theorem 4.7 for which $|W|=\lambda$. For any $x \in W$ define the set $\eta(x)=\{y \in W \mid(x, y) \in R\}$. It is plain to see that $\eta(x) \neq \varnothing$ for any $x \in W$. Let

$$
B=W \times \lambda .
$$

By $\vec{a}^{C} \in T B$ where $C \subseteq W$ we denote an isomorphic copy of $\vec{a}$ for which the set $\{x\} \times \lambda$ is $\mathrm{Flt}_{B}\left(\vec{a}^{C}\right)$-stationary if and only if $x \in C$. A construction of $\vec{a}^{C}$ is done by taking an injective mapping $i_{C}: A \rightarrow W \times \lambda$ for which the elements of the partition $\left\{A_{k}\right\}_{k \leq \lambda}$ of $A$ are mapped into subsets $\{x\} \times \lambda$ for $x \in C$ and taking $\vec{a}^{C}:=T i_{C}(\vec{a})$. Such an injective mapping exists by the fact that any subset $C \subseteq W$ has cardinality at most $\lambda$. Let $\vec{a}_{B, 1} \in T B$ be defined as $\vec{a}_{B, 1}:=\vec{a}^{W}$. Finally, choose $k \in \lambda$ and let $\vec{a}_{B, 2}$ be an isomorphic copy of $\vec{a}$ such that $\vec{a}_{B, 2} \in T[W \times\{k\}] \subset T B$.

Now we are ready to define the structure $\beta: B \rightarrow T B$ of the coalgebra $\mathbb{B}$ :

$$
\beta(v):=\vec{a}^{\eta(x)} \text { for } v \in\{x\} \times \lambda .
$$

Lemma 4.8. Given a homomorphism $h: \mathbb{S} \rightarrow \mathbb{B}$ where $\mathbb{S} \leq \mathbb{B}$, for any $x \in W$ we have $h(\{x\} \times \lambda \cap S) \subseteq\{y\} \times \lambda$ for some $y \in W$.

Proof. If there were two elements $\left(x, l_{1}\right) \in S$ and $\left(x, l_{2}\right) \in S$ such that $h\left(x, l_{1}\right) \in$ $\{y\} \times \lambda$ and $h\left(x, l_{2}\right) \in\{z\} \times \lambda$, then since $\beta\left(x, l_{1}\right)=\beta\left(x, l_{2}\right)$ we would have $T h\left(\beta\left(x, l_{1}\right)\right)=\beta\left(h\left(x, l_{1}\right)\right)=\beta\left(h\left(x, l_{2}\right)\right)=T h\left(\beta\left(x, l_{2}\right)\right)$ and therefore, $\vec{a}^{\eta(y)}=$ $\vec{a}^{\eta(z)}$. If $y \neq z$, then it means that in $(W, R)$ two distinct elements $y$ and $z$ have the same family of neighbours, i.e., $\eta(y)=\eta(z)$. This implies that a function $f: W \rightarrow W$ which is defined as $f(x)=x$ for $x \notin\{y, z\}, f(z)=y$ and $f(y)=z$ is a homomorphism from the binary relation $(W, R)$ into $(W, R)$ which is not an identity. This contradicts the fact that $(W, R)$ is rigid. 
Lemma 4.9. If $\mathbb{S} \leq \mathbb{B}$ is a subcoalgebra with $S \cap\{x\} \times \lambda \neq \varnothing$ for any $x \in W$ and $h: \mathbb{S} \rightarrow \mathbb{B}$ is a homomorphism, then

$$
h(\{x\} \times \lambda \cap S) \subseteq\{x\} \times \lambda \text { for any } x \in W .
$$

Proof. By Lemma 4.8, given a homomorphism $h: \mathbb{S} \rightarrow \mathbb{B}$ for any element $x \in W$ we have $h(\{x\} \times \lambda \cap S) \subseteq\{y\} \times \lambda$ for some $y \in W$. Given a pair $(x, l) \in S$ we have

$$
T h\left(\vec{a}^{\eta(x)}\right)=T h(\beta(x, l))=\beta(h(x, l))=\beta\left(\left(y, l^{\prime}\right)\right)=\vec{a}^{\eta(y)} .
$$

This means that

$$
\mathcal{F} h\left(\mathrm{Flt}_{S}\left(\vec{a}^{\eta(x)}\right)\right)=\left\{W \mid h(U) \subseteq W \text { for } U \in \mathrm{Flt}_{S}\left(\vec{a}^{\eta(x)}\right)\right\}=\mathrm{Flt}_{B}\left(\vec{a}^{\eta(y)}\right) .
$$

By the equality above and by the fact that the set $\eta(x) \times \lambda \cap S$ is $\operatorname{Flt}_{S}\left(\vec{a}^{\eta(x)}\right)$ stationary and the sets $\{t\} \times \lambda$ for $t \in \eta(y)$ are Flt $_{B}\left(\vec{a}^{\eta(y)}\right)$-stationary we have

$$
\begin{gathered}
h(\eta(x) \times \lambda \cap S) \subseteq \eta(y) \times \lambda, \text { and } \\
h(\eta(x) \times \lambda \cap S) \cap\{z\} \times \lambda \neq \varnothing \text { for any } z \in \eta(y) .
\end{gathered}
$$

This exactly means that if we define $\tilde{h}: W \rightarrow W$ as $\tilde{h}(x)=\pi_{1}(h((x, k)))$ for any $k \in \lambda$, then $\tilde{h}(\eta(x))=\eta(\tilde{h}(x))$. Therefore, $\tilde{h}: W \rightarrow W$ is a homomorphism from $(W, B)$ to itself. Since $(B, W)$ is rigid this implies that $\tilde{h}=i d_{W}$. Thus, for $(x, l) \in\{x\} \times \lambda \cap S$ we have $h(x, l)=\left(x, l^{\prime}\right)$ for some $l^{\prime} \in \lambda$.

We will show that $\langle B, \beta\rangle, \vec{a}_{B, 1}$ and $\vec{a}_{B, 2}$ satisfy properties (1) and (2) listed at the beginning of this section. It is clear that the property (1) is satisfied. To show (2) is true choose a subcoalgebra $\mathbb{S}$ of $\mathbb{B}$ with $\vec{a}_{B, 1}, \vec{a}_{B, 2} \in T S$ and a homomorphism $h: \mathbb{S} \rightarrow \mathbb{C}$ where $\mathbb{C}$ has $\mathbb{B}$ as its subcoalgebra, $\operatorname{Th}\left(\vec{a}_{B, 1}\right)=$ $\vec{a}_{B, 1} \in T B \subseteq T C$ and $\gamma(C \backslash B)=\left\{\vec{a}_{B, 1}, \perp\right\}$. By the definition of $\vec{a}_{B, 1}=\vec{a}^{W}$ and by the fact $\vec{a}_{B, 1}=\vec{a}^{W} \in T S$ it follows that $S \cap\{x\} \times \lambda \neq \varnothing$ for any $x \in W$. The equality $\operatorname{Th}\left(\vec{a}_{B, 1}\right)=\operatorname{Th}\left(\vec{a}^{W}\right)=\vec{a}_{B, 1}=\vec{a}^{W}$ implies that

$$
\mathcal{F} h\left(\mathrm{Flt}_{S}\left(\vec{a}^{W}\right)\right)=\left\{W \mid h(U) \subseteq W \text { and } U \in \mathrm{Flt}_{S}\left(\vec{a}^{W}\right)\right\}=\mathrm{Flt}_{C}\left(\vec{a}^{W}\right) .
$$

Any set $U \in \mathrm{Flt}_{S}\left(\vec{a}^{W}\right)$ intersects with $\{x\} \times \lambda$ for all $x \in W$. That is why $\beta(U)=\left\{\vec{a}^{\eta(x)} \mid x \in W\right\}=\beta(S)$. Moreover, for any $U \in \mathrm{Flt}_{S}\left(\vec{a}^{W}\right)$ the set $h(U) \in \mathrm{Flt}_{C}\left(\vec{a}^{W}\right)$ intersects with any $\{x\} \times \lambda \subset C$ for $x \in W$ and therefore, $\gamma(h(U)) \supseteq \gamma(B)=\beta(B)=\left\{\vec{a}^{\eta(x)} \mid x \in W\right\}$.

Since $B \in \mathrm{Flt}_{C}\left(\vec{a}^{W}\right)$, there is $V \in \mathrm{Flt}_{S}\left(\vec{a}^{W}\right)$ such that $h(V) \subseteq B$. Therefore, $T h(\beta(S))=T h(\beta(V))=\beta(h(V))=\beta(B)$. By the equality $\gamma(C \backslash B)=$ $\left\{\vec{a}_{B, 1}, \perp\right\}$ we have $h(S) \subseteq B$.

In other words, we may treat the homomorphism $h: \mathbb{S} \rightarrow \mathbb{C}$ as a homomorphism from $\mathbb{S}$ to $\mathbb{B}$.

We will now show that $T h\left(\vec{a}_{B, 2}\right) \notin \beta(B) \cup\left\{\vec{a}_{B, 1}, \perp\right\}$. First of all, we will show $T h\left(\vec{a}_{B, 2}\right) \notin \beta(B) \cup\left\{\vec{a}_{B, 1}\right\}$. By contradiction assume that there is $C \subseteq W$ such that $T h\left(\vec{a}_{B, 2}\right)=\vec{a}^{C}$. Therefore,

$$
\mathcal{F} h\left(\mathrm{Flt}_{S}\left(\vec{a}_{B, 2}\right)\right)=\left\{W \mid h(U) \subseteq W \text { and } U \in \mathrm{Flt}_{S}\left(\vec{a}_{B, 2}\right)\right\}=\mathrm{Flt}_{C}\left(\vec{a}^{C}\right) .
$$


Hence, there is $U \in \mathrm{Flt}_{S}\left(\vec{a}_{B, 2}\right)$ such that

- $U \subseteq W \times\{k\}$,

- $h(U) \subseteq B$,

- $h(U)$ intersects with $\{x\} \times \lambda$ for any $x \in C$.

Fix any $x \in C$. The set $[\{x\} \times \lambda] \cap h(U)$ has cardinality $\lambda$. This means that there is $V \subseteq U$ of cardinality $\lambda$ such that $h(V) \subseteq\{x\} \times \lambda$. Therefore, there are two distinct elements $(y, k),(z, k) \in V$ such that $h(y, k) \in\{x\} \times \lambda$ and $h(z, k) \in\{x\} \times \lambda$. By Lemma 4.9 this is a contradiction. Therefore, $T h\left(\vec{a}_{B, 2}\right) \notin \beta(B) \cup\left\{\vec{a}_{B, 1}\right\}$.

Now it is enough to show that $\operatorname{Th}\left(\vec{a}_{B, 2}\right) \neq \perp$. This is easy to see because by Lemma 4.9 the restriction $h_{\mid W \times\{k\}}: W \times\{k\} \rightarrow B$ of $h$ is a one-to-one mapping. Thus, $T h\left(\vec{a}_{B, 2}\right)=T h_{\mid W \times\{k\}}\left(\vec{a}_{B, 2}\right) \neq \perp$. Hence, the coalgebra $\mathbb{B}$ and elements $\vec{a}_{B, 1}, \vec{a}_{B, 2}$ satisfy property (2).

Theorem 4.10. Let $T:$ Set $\rightarrow$ Set be a connected functor that preserves non-empty preimages on undistinguished points and $\mathcal{C}_{0,1}<T$. Let there be a set $A$ of uncountable cardinality $\lambda$ together with its partition $\left\{A_{l}\right\}_{l \leq \lambda}$ and an undistinguished point $\vec{a} \in T A$ for which any element $A_{l}$ of the partition of $A$ is $\mathrm{Flt}_{A}(\vec{a})$-stationary. Then there exists a T-coalgebra $\mathbb{X}$ such that $\mathcal{H} \mathcal{S}(\mathbb{X}) \neq \mathcal{S H}(\mathbb{X})$

\subsection{Subfunctors of factor functors of the Stone-Czech functor $\mathcal{B}$.} Let $T, \perp, A, \vec{a} \in T A, \lambda$ be as in the Standing Hypothesis. Let us recall that $\mathcal{B}:$ Set $\rightarrow$ Set is the functor sending any set $X$ to the underlying set of the Stone-Czech compactification $\mathcal{B} X$ of $X$ viewed as a discrete space and, for $f: X \rightarrow Y, \mathcal{B} f: \mathcal{B} X \rightarrow \mathcal{B} Y$ is the underlying map of the continuous extension of $f$. The functor $\mathcal{B}$ can be also described as follows: it is a subfunctor of the functor $\mathcal{F}$ with $\mathcal{B} X$ being the set of all ultrafilters on $X$. Since $\mathcal{B}$ preserves all preimages, there is no problem with $\mathcal{B}$-coalgebras, i.e., $\mathcal{H} \mathcal{S}=\mathcal{S H}$ is valid for them.

The functor $\mathcal{B}$ is connected and faithful, the copy of $\mathcal{I} d$ in $\mathcal{B}$ is formed by all fixed ultrafilters (ultrafilters with non-empty intersections).

Let $\nu: \mathcal{B} \rightarrow \overline{\mathcal{B}}$ be an epitransformation which glues together all fixed ultrafilters on any set $X$ into one element of $\overline{\mathcal{B}} X$ - the distinguished point $\perp \in \overline{\mathcal{B}} X$. The functor $\overline{\mathcal{B}}$ does not preserve non-empty preimages. For $\overline{\mathcal{B}}$-coalgebras we get $\mathcal{H S} \neq \mathcal{S H}$ simply by applying the construction presented in Subsection 4.3: put $\overline{\mathcal{B}} \omega=T A$ and let $\vec{a}$ be any free ultrafilter on $\omega$.

Interesting subfunctors $\overline{\mathcal{B}}_{M}$ of $\overline{\mathcal{B}}$ are determined by a fixed subset $M \subseteq \overline{\mathcal{B}} X$ as follows:

$$
\overline{\mathcal{B}}_{M}(Y)=\{\perp\} \cup\{[\overline{\mathcal{B}} f](\mathcal{U}) \mid \mathcal{U} \in M \text { and } f: X \rightarrow Y\} .
$$

We already know that $\mathcal{H} \mathcal{S} \neq \mathcal{S H}$ for $\overline{\mathcal{B}}_{M}$-coalgebras in the case when $M$ contains an ultrafilter on $\omega$. 
We were not able to decide whether $\mathcal{H} \mathcal{S} \neq \mathcal{S H}$ for $\overline{\mathcal{B}}_{M}$-coalgebras whenever $M$ consists of exactly one ultrafilter on arbitrarily large set $X$. This problem remains open.

On the other hand, $\mathcal{H S} \neq \mathcal{S H}$ can be shown for $\overline{\mathcal{B}}_{M}$-coalgebras if $M$ consists of ultrafilters on an arbitrarily large set $X$ whenever the set $M$ itself is "sufficiently rich" which will be explained below. We recall that a Rudin-Keisler order on ultrafilters is defined as follows: for $\mathcal{U} \in \mathcal{B} X$ and $\mathcal{V} \in \mathcal{B} Y$, we write $\mathcal{U} \leq \mathcal{V}$ if and only if there exists $f: Y \rightarrow X$ such that $\mathcal{B} f(\mathcal{V})=\mathcal{U}$. We will use the fascinating result of Shelah and Rudin [6]:

for any infinite cardinal $\kappa$ there exist $2^{2^{\kappa}}$ ultrafilters on a set $X$ with $|X|=\kappa$ which are pairwise incomparable in the Rudin-Keisler order.

By the above result we know that we are able to construct a family $M \subset \overline{\mathcal{B}} A$ of ultrafilters on arbitrary large $A$ which will contain at least $|A|$-many incomparable ultrafilters in the Rudin-Keisler order. Now, if we assume that $M$ possesses this property, then we will be able to show that $\mathcal{H} \mathcal{S} \neq \mathcal{S H}$. Indeed, we will now construct a $\overline{\mathcal{B}}_{M}$-coalgebra $\mathbb{B}$ and elements $\vec{a}_{B, 1}$ and $\vec{a}_{B, 2}$ satisfying properties (1) and (2) from the Standing Hypothesis. Let $B=A$. Therefore, $|B|=|A|=\lambda$. For any $v \in B$ define $\vec{a}_{v} \in \overline{\mathcal{B}}_{M} B$ so that the family $\left\{\vec{a}_{v}\right\}_{v \in B}$ is a family of incomparable ultrafilters. Introduce incomparable $\vec{a}_{B, 1}, \vec{a}_{B, 2} \in \overline{\mathcal{B}}_{M} B$ such that both ultrafilters are incomparable with $\vec{a}_{v}$ for any $v \in B$. Finally, define $\beta: B \rightarrow \overline{\mathcal{B}}_{M} B ; v \mapsto \vec{a}_{v}$. It is clear that $\mathbb{B}=\langle B, \beta\rangle, \vec{a}_{B, 1}$ and $\vec{a}_{B, 2}$ satisfy property (1). We will show that they also satisfy (2). In order to do this take the coalgebras $\mathbb{S}$ and $\mathbb{C}=\langle C, \gamma\rangle$ such that $\mathbb{S} \leq \mathbb{B} \leq \mathbb{C}$ with $\vec{a}_{B, 1}, \vec{a}_{B, 2} \in \overline{\mathcal{B}}_{M} S$ and $\gamma(C \backslash B)=\left\{\vec{a}_{B, 1}, \perp\right\}$. Since for different $v, w \in S$ the filters $\vec{a}_{v}$ and $\vec{a}_{w}$ are incomparable, then any homomorphism $h: \mathbb{S} \rightarrow \mathbb{C}$ is a one-to-one mapping. Hence, $T h$ is one-to-one and $T h\left(\vec{a}_{B, 2}\right) \neq \perp$.

Since the Rudin-Keisler order may be generalized to any filters, the above result may also be easily generalized to the following one.

Theorem 4.11. Let $T:$ Set $\rightarrow$ Set be a connected functor that preserves nonempty preimages on undistinguished points and $\mathcal{C}_{0,1}<T$. Let there be a set $A$ of cardinality $\lambda$ and let there be a family $\left\{\vec{a}_{l}\right\}_{l \in \lambda} \subseteq T A$ for which the filters Flt $_{A}\left(\vec{a}_{l_{1}}\right)$ and Flt $_{A}\left(\vec{a}_{l_{2}}\right)$ are incomparable in the generalization of the RudinKeisler order for any $l_{1} \neq l_{2}$. Then there exists a $T$-coalgebra $\mathbb{X}$ such that $\mathcal{H} \mathcal{S}(\mathbb{X}) \neq \mathcal{S H}(\mathbb{X})$.

\section{REFERENCES}

[1] Adamék, J., Trnková, V.: Automata and Algebras in a Category. Kluwer, Dordrecht (1990)

[2] Gumm, H.P.: Elements of the general theory of coalgebras. LUATCS 99, Rand Afrikaans University, Johannesburg (1999)

[3] Gumm, H.P.: From T-coalgebras to filter structures and transtion systems. In: Algebra and Coalgebra in Computer Science. Lecture Notes in Computer Science, vol. 3629. Springer (2005)

[4] Gumm, H.P., Schröder, T.: Types and coalgebraic structure. Algebra Universalis 53, $229-252(2005)$ 
[5] Pultr, A., Trnková, V.: Combinatorial, Algebraic and Topological Representations of Groups, Semigroups and Categories. North Holland (1980). Also: Academia Praque (1980)

[6] Shelah, S., Rudin, M.E.: Unordered types of ultrafilters. Topology Proc. 3, 199-204 (1978)

[7] Trnková, V.: Some properties of Set-endofunctors. Comment. Math. Univ. Carolin. 10, 323-259 (1969)

[8] Trnková, V.: On descriptive classification of set-functors I. Comment. Math. Univ. Carolin. 12 (1971)

[9] Vopěnka, P., Pultr, A., Hedrlín, Z.: A rigid relation exists on any set. Comment. Math. Univ. Carolin. 6, 149-155 (1965)

[10] Zmrzlina, A.: Too Many Functors - A continuation of "The Emergence of Functors". In: Categorical Perpectives. Trends in Mathematics, pp. 47-62. Birkhauser (2001)

\section{TOMASZ BREngos}

Faculty of Mathematics and Information Sciences, Warsaw University of Technology, Pl. Politechniki 1, 00-661 Warszawa, Poland

e-mail: t.brengos@mini.pw.edu.pl

\section{VĚra Trnková}

Math. Institute of Charles University, Sokolovska 83, 18675 Praha 8, Czech Republic e-mail: trnkova@karlin.mff.cuni.cz

Open Access This article is distributed under the terms of the Creative Commons Attribution Noncommercial License which permits any noncommercial use, distribution, and reproduction in any medium, provided the original author(s) and source are credited. 